\title{
Simulation of Spin Transport Properties in Schottky Barrier FET Using Monte Carlo Method
}

\author{
Jianhua Liu ${ }^{\text {la }}$, Gang Du, Ji Cao, Zhiliang Xia, Yi Wang, Ruqi Han, Xiaoyan Liu ${ }^{\text {lb }}$ \\ Institute of Microelectronics, Peking University, Beijing 100871, China \\ aliujianhua@ime.pku.edu.cn「xyliu@ime.pku.edu.cn
}

\begin{abstract}
We simulate the spin transport properties in Schottky Barrier FET by ensemble Monte Carlo Method. Based on the three subbands approximations of 2DEG a more accurate model to calculate the spin precession frequency is adopted. With intra-subband and inter-subband scatterings fully considered, the three subbands approximation is compared with the single band approximation. We also examine the influence of the external electric field on the dephasing of the injected spin polarization. The simulation results can provide some guidance for the future design of SpinFET.
\end{abstract}

\section{Introduction}

Spin FET was first proposed by Datta and Das in 1990[1]. The most straightfoward way for spin injection is to form an ohmic contact between an FM and a semiconductor surface. However, this way has two intrinsic shortcomings. One is that the heavily doped semiconductor will lead to spin-flip scattering. The other, pointed by Schmidt et al.[2], is that conductivity mismatching between ferromagnetic and semiconductor will decrease the spin injection effectiveness dramatically. Rashba and Flatte[3] theoretically proved that tunnel barrier will provide a way to overcome the conductivity mismatch problem. So Schottky Barrier FET is a structure suitable for spin current injection. Some research groups have simulated this structure by Monte Carlo Method [4,5]. However, scatterings which are of great importance have not been fully considered. Using ensemble Monte Carlo method we investigate the spin transport properties with scattering treated carefully in the Schottky Barrier FET.

\section{Model Descriptions}

Fig. 1 shows the schematic device structure and the coordinate system we used. The potential profile along y: $V(y)=-V_{0} \exp \left(-y / Y_{0}\right), y>0 ; \quad V(y)=\infty, y<0$ is adopted[6]. By using variational method we get analytical wave function of the three lowest subbands[7]

$$
\begin{aligned}
& \psi_{1}(y)=K_{1} y \exp \left(\frac{-c_{1} y}{2}\right), \psi_{2}(y)=K_{2}\left(y+a_{2} y^{2}\right) \exp \left(\frac{-c_{2} y}{2}\right) . \\
& \psi_{3}(y)=K_{3}\left(y+a_{3} y^{2}+b_{3} y^{3}\right) \exp \left(\frac{-c_{3} y}{2}\right)
\end{aligned}
$$




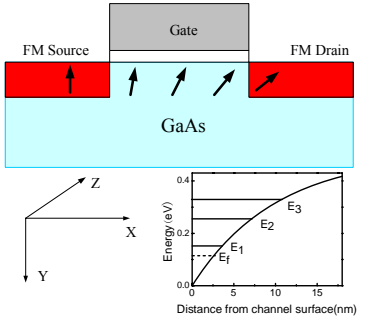

Fig.1. Schematic device structure of SpinFET and three sub-bands model

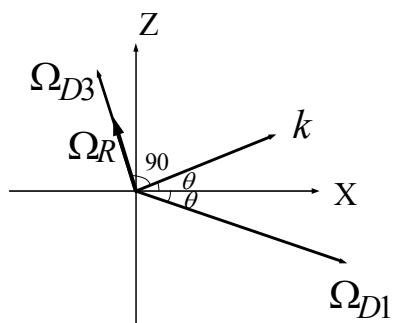

Fig.2. Three components of the spin precessing vector

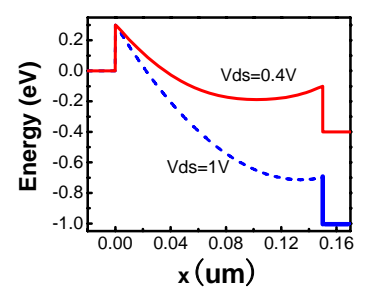

Fig.3. Potential profile along the channel at $\mathrm{Vds}=0.4 \mathrm{~V}$ and $\mathrm{Vds}=1.0 \mathrm{~V}$

The two dimensional scattering rates are evaluated as follows:[8]

$W_{k, a c}=\frac{m \Xi_{0}^{2} k_{B} T}{2 \pi \hbar^{3} c_{l}} \phi_{n m}, \phi_{n m}=2 \pi \int_{0}^{\infty} \psi_{n}^{2}(y) \psi_{m}^{2}(y) d z, W_{k, p o p}=\frac{e^{2} \omega_{0}}{8 \pi^{2} \varepsilon_{p}}\left(n_{\omega_{0}}+\frac{1}{2} \mp \frac{1}{2}\right) \frac{k}{E_{(k)}} \int_{q_{\min }}^{q_{\max }} \frac{F_{n, m(q)}}{\sin \theta} d q$

$F_{n, m(q)}=\int_{-\infty}^{\infty} \frac{1}{q^{2}+q_{z}^{2}}\left|G_{n, m\left(q_{z}\right)}\right|^{2} d q_{z}, G_{n, m\left(q_{z}\right)}=\int_{0}^{\infty} \psi_{n(z)} e^{i q_{z}} \psi_{m(z)} d z$

$\mathrm{W}_{\mathrm{k}, \mathrm{ac}}$ and $\mathrm{W}_{\mathrm{k}, \mathrm{pop}}$ represent acoustic phonon scattering rate and polar optical phonon scattering rate respectively.

In III-V semiconductor, D'yakonov-Perel' mechanism is the dominant spin dephasing mechanism. Under its influence, the spin precessing process is described as follow:

$\frac{d \vec{S}}{d t}=\vec{\Omega} \times \vec{S}, \vec{\Omega}=\vec{\Omega}_{R}+\vec{\Omega}_{D 1}+\vec{\Omega}_{D 3}$, where

$\bar{\Omega}_{R}=\frac{2 a_{46} E_{y}}{\hbar}\left(-k_{z} \bar{u}_{x}+k_{x} \bar{u}_{z}\right), \quad \bar{\Omega}_{D 1}=\frac{2 a_{42}}{\hbar}\left\langle k_{y}^{2}\right\rangle\left(k_{x} \bar{u}_{x}-k_{z} \bar{u}_{z}\right), \quad \bar{\Omega}_{D 3}=\frac{2 a_{42}}{\hbar}\left(-k_{x} k_{z}^{2} \bar{u}_{x}+k_{z} k_{x}^{2} \bar{u}_{z}\right)$

They correspond to the Rashba term[9], Dresselhaus[10]linear term and cubic term respectively. $\mathrm{a}_{46}$ and $\mathrm{a}_{42}$ are material parameters. Fig. 2 shows the three components of $\vec{\Omega}$. $\left\langle k_{y}^{2}\right\rangle$ is the average of the operator $-(\partial / \partial y)^{2}$ over the wave functions. In some works[7,11,12], the potential vertical to the channel are treated as quantum well or triangular well, and hence the $\left\langle k_{y}^{2}\right\rangle$ is not accurate enough. Our simulation provides a more accurate model of $\left\langle k_{y}^{2}\right\rangle$ based on the wavefunctions of three subbands:

$$
\begin{aligned}
& \left\langle k_{y 1}^{2}\right\rangle=-\int_{0}^{\infty} \Psi_{1} \frac{\partial^{2} \Psi_{1}}{\partial y^{2}} d y=\frac{\hbar^{2} K_{1}{ }^{2}}{2 c_{1}} \quad\left\langle k_{y 2}^{2}\right\rangle=-\int_{0}^{\infty} \Psi_{2} \frac{\partial^{2} \Psi_{2}}{\partial y^{2}} d y=\hbar^{2} K_{2}{ }^{2} \frac{4 a_{2}{ }^{2}+2 a_{2} c_{2}+c_{2}{ }^{2}}{2 c_{2}{ }^{3}} \\
& \left\langle k_{y 3}^{2}\right\rangle=-\int_{0}^{\infty} \Psi_{3} \frac{\partial^{2} \Psi_{3}}{\partial y^{2}} d y=\hbar^{2} K_{3}{ }^{2} \frac{72 b_{3}{ }^{2}+24 a_{3} b_{3} c_{3}+4 a_{3}{ }^{2} c_{3}{ }^{2}+2 a_{3} c_{3}{ }^{3}+c_{3}{ }^{4}}{2 c_{3}{ }^{5}}
\end{aligned}
$$

\section{Simulation Results and Discussions}

Fig.3 shows the potential distribution along the channel in SB FET. Fig.4. shows spin dephasing process of an electron without scattering and electrical field. Fig. 5,6,7 plot 
spin polarization distribution along the channel of the $150 \mathrm{~nm}$ gate length SB FET with the initial injection spin directions along $\mathrm{x}, \mathrm{y}, \mathrm{z}$ axis respectively. It's can be seen that the dephasing rate is different under different injection orientation, for example, it is lowest when injected along $\mathrm{x}$ axes.

In order to compare the influence of scattering on the spin transport, the ratio of $|\mathrm{S}|=\sqrt{S_{x}^{2}+S_{y}^{2}+S_{z}^{2}}$ in the drain and source side is used and referred as $|\mathrm{S}|_{\mathrm{D}} /|\mathrm{S}|_{\mathrm{S}}$ here. The reduction of this ratio corresponds to initial spin polarization dephasing as electrons transport from source to drain. Fig. 8 displays the comparison between the simulation results with scattering included or not for various Vds. From the figure, it can be seen that $|\mathrm{S}|_{\mathrm{D}} /|\mathrm{S}|_{\mathrm{S}}$ reduces more rapidly when scattering is included. While Vds increasing, the average precessing frequency becomes quicker and consequently spin dephasing rate becomes faster. Fig. 9 demonstrates the differences of $|\mathrm{S}|_{\mathrm{D}} /|\mathrm{S}|_{\mathrm{S}}$ between the three- subbands and single band approximations. Inter-subband scattering plays an important role in the former. It can be seen that at small Vds the two approximations are of considerable differences.

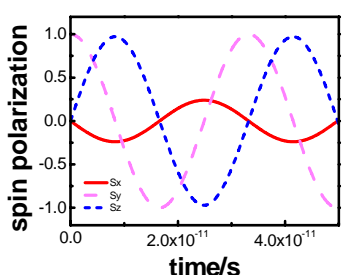

Fig.4. Illustration of single Fig.5. Spin polarization electron's spin precessing distribution with injection process without scattering and direction along $\mathrm{x}$ axis at electrical field

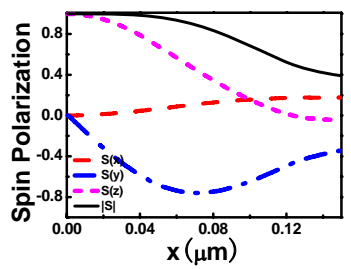

Fig.7. Spin polarization distribution with injection direction along $\mathrm{z}$ axis at $\mathrm{Vds}=1.0 \mathrm{~V}$

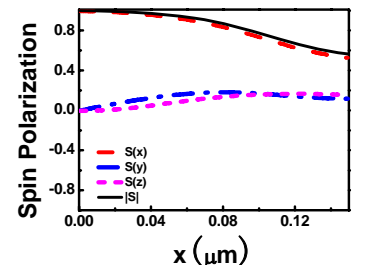

$\mathrm{Vds}=1.0 \mathrm{~V}$

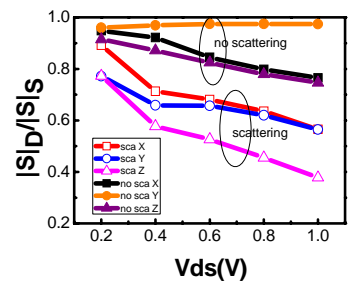

Fig. $8 . \quad$ Comparison of
$\mathrm{S} \mid$ considering scattering and without scattering

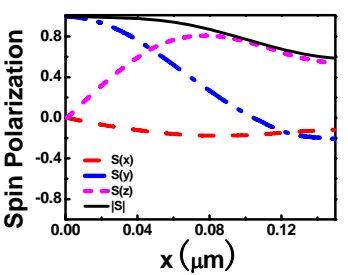

Fig.6. Spin polarization distribution with injection direction along $y$ axis at $\mathrm{Vds}=1.0 \mathrm{~V}$

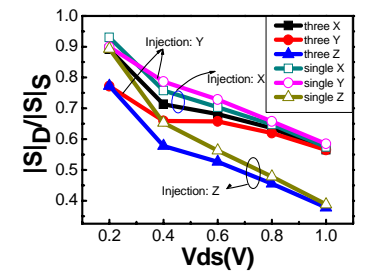

Fig.9. comparison of $\left.S\right|_{D} /|S|_{S}$ between the three subbands approximation and single subband approximation

Fig. 10 illustrates the influence of Rashba and Dresselhaus term and shows that Dresselhaus effect is dominant. This conclusion is suitable for GaAs but not all semiconductors because for narrow gap semiconductor such as $\mathrm{InSb} \mathrm{a}_{42}$ is much larger than GaAs and then Rashba effect also can exert an important influence. Fig.11 plots 
the spin polarization distribution with gate length $350 \mathrm{~nm}$. It can be seen that the three components of $\mathbf{S}$ have all decreased to nearly zero. Fig.12 displays the spin dephasing rates with different channel length. The results indicate that $|\mathrm{S}|_{\mathrm{D}} /|\mathrm{S}|_{\mathrm{S}}$ reduces with channel length increasing and thus channel length of the spin FET made by GaAs should not be too large for the conservation of spin current.

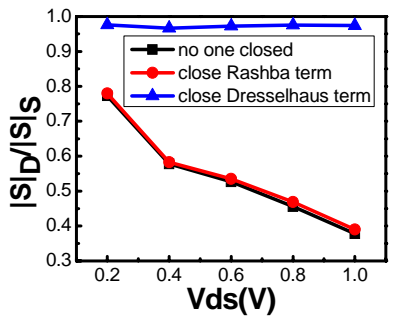

Fig.10. Influence of Rashba term and Dresselhaus term on the spin transport in $\mathrm{SB}$ FET.

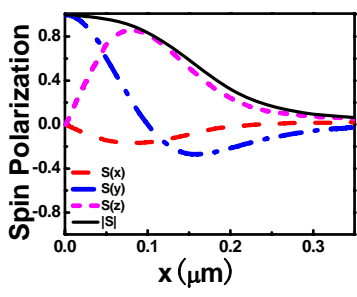

Fig.11. Spin polarization distribution with injection along $\mathrm{y}$ axis at $\mathrm{Vds}=1.0 \mathrm{~V}$ with $350 \mathrm{~nm}$ gate length.

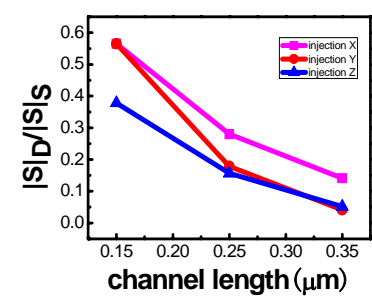

Fig.12. spin dephasing rate for electrons transport across SB FET with channel length from $150 \mathrm{~nm}$ to $350 \mathrm{~nm}$.

\section{Conclusions}

Using the Ensemble Monte Carlo Method, we simulated the spin transport properties of electrons in the Schottky-Barrier FET. Scattering mechanisms including the intra-subband scattering and inter-subband scattering are considered. The influence of $\mathrm{V}_{\mathrm{ds}}$ and the orientation of the injected spin current are investigated. We also compare the single subband and three subbands approximations and find that the results are of considerable difference. Finally, the influence of channel length shows that nano scale gate length is necessary for the SB spinFET.

\section{Acknowledgement}

This work is supported by NKBRP 2006CB302705 and NSFC 60606013.

\section{References}

[1] S. Datta and B. Das, Appl. Phys. Lett. 56, 665 (1990).

[2] G. Schmidt, et.al, Phys. Rev. B 62, R4790 (2000).

[3] E.I. Rashba, Phys. Rev. B 62, 16267 (2000).

[4] Min Shen, et.al, J. App. Phys. 96, 4319(2004)

[5] Y. Y. Wang ,et.al, Phys. Rev. B, 72, 153301(2005)

[6] K. Tomizawa, N. Hashizume, IEEE T. Electron Dev. 35 849(1988)

[7] L.G. Kong, et.at, SISPAD 2005, 175-178

[8] J.Price, Surf. Sci. ,113, 199 (1982)

[9] E. I. Rashba, Sov. Phys. Semicond. 2, 1109 (1960)

[10] G. Dresselhaus, Phys. Rev. 100, 580 (1955)

[11] [16] S. Pramanik, S. Bandyopadhyay, Phys. Rev. B 68 075313(2003)

[12] Rogerio de Sousa and S. Das Sarma, Phys. Rev. B 68, 155330(2003) 